\title{
A NOTE ON A RECENT PAPER BY ZAKS, FROSTIG AND LEVIKSON
}

\author{
BY \\ KLAus D. SCHMIDT
}

\begin{abstract}
In the present paper we give a short proof of a result of Zaks, Frostig and Levikson [2006] on the solution of an optimization problem which is related to the problem of optimal pricing of a heterogeneous portfolio.
\end{abstract}

Following Zaks, Frostig and Levikson [2006], we consider a heterogeneous portfolio which is composed by $k$ risk classes such that for each $j \in\{1, \ldots, k\}$ the risk class $j$ contains $n_{j}$ risks $X_{j, 1}, \ldots, X_{j, n_{j}}$ which are assumed to be i.i.d. with finite first and second moments and non-zero variance. Then the total risk of risk class $j$ is defined as

$$
S_{j}:=\sum_{i=1}^{n_{j}} X_{j, i}
$$

Consider also $r_{1}, \ldots, r_{k} \in(0, \infty)$ and $\alpha \in(0,1)$, and let $z_{1-\alpha}$ denote the $1-\alpha$ percentile of the standard normal distribution. The authors prove the following result:

Theorem 1. The minimization problem

Minimize

$$
\sum_{j=1}^{k}\left(\frac{1}{r_{j}} E\left[\left(S_{j}-n_{j} \pi_{j}\right)^{2}\right]\right)
$$

over $\pi_{1}, \ldots, \pi_{k}$ subject to

$$
\sum_{j=1}^{k} n_{j} \pi_{j}=E\left[\sum_{j=1}^{k} S_{j}\right]+z_{1-\alpha} \sqrt{\operatorname{var}\left[\sum_{j=1}^{k} S_{j}\right]}
$$

has a unique solution $\pi_{1}^{*}, \ldots, \pi_{k}^{*}$ and the identity 


$$
\pi_{j}^{*}=\frac{1}{n_{j}}\left(E\left[S_{j}\right]+\frac{r_{j}}{\sum_{i=1}^{k} r_{i}} z_{1-\alpha} \sqrt{\operatorname{var}\left[\sum_{i=1}^{k} S_{i}\right]}\right)
$$

holds for all $j \in\{1, \ldots, k\}$.

Let now $\mathbf{S}$ denote the random vector with coordinates $S_{1}, \ldots, S_{k}$ and let $\boldsymbol{v}:=$ $E[\mathbf{S}]$. Let also $\mathbf{V}$ denote the diagonal matrix with diagonal elements $r_{1}, \ldots, r_{k}$, let $\mathbf{1}$ denote the vector with all coordinates being equal to one, and consider $t \in \mathbb{R}$. Using this notation, Theorem 1 can be stated in the following form, which suggests a simple proof based on the projection theorem in Hilbert spaces (see e.g. De Vylder [1996; Part III] or Swartz [1994; Section 6.6]):

Theorem 1'. The minimization problem

Minimize

$$
E\left[(\mathbf{S}-\mathbf{p})^{\prime} \mathbf{V}^{-1}(\mathbf{S}-\mathbf{p})\right]
$$

over $\mathbf{p}$ subject to $\mathbf{1}^{\prime} \mathbf{p}=\mathbf{1}^{\prime} \mathbf{v}+t$

has a unique solution $\mathbf{p}^{*}$ and the solution satisfies $\mathbf{p}^{*}=\boldsymbol{v}+t\left(\mathbf{1}^{\prime} \mathbf{V} \mathbf{1}\right)^{-1} \mathbf{V} \mathbf{1}$.

Proof. Since the matrix $\mathbf{V}$ is symmetric and positive definite, the vector space $L^{2}\left(\mathbb{R}^{k}\right)$ consisting of all $k$-dimensional random vectors having finite second moments is a Hilbert space under the inner product $\langle., .\rangle_{\mathbf{V}}$ given by

$$
\langle\mathbf{X}, \mathbf{Y}\rangle_{\mathbf{V}}:=E\left[\mathbf{X}^{\prime} \mathbf{V}^{-1} \mathbf{Y}\right]
$$

and the induced norm $\|\cdot\|_{\mathbf{V}}$ given by

$$
\|\mathbf{X}\|_{\mathbf{V}}:=\langle\mathbf{X}, \mathbf{X}\rangle_{\mathbf{V}}^{1 / 2}
$$

(Here, as usual, two random vectors $\mathbf{X}, \mathbf{Y}$ are identified if $P[\{\mathbf{X}=\mathbf{Y}\}]=1$.) Furthermore, the set

$$
A:=\left\{\mathbf{p} \in \mathbb{R}^{k} \mid \mathbf{1}^{\prime} \mathbf{p}=\mathbf{1}^{\prime} \boldsymbol{v}+t\right\}
$$

is a nonempty closed subset of $L^{2}\left(\mathbb{R}^{k}\right)$. Since $A$ is convex, it follows from the projection theorem in Hilbert spaces that the minimization problem

Minimize

$$
\|S-\mathbf{p}\|_{\mathbf{v}}
$$

over $\mathbf{p} \in A$ 
has a unique solution $\mathbf{p}^{*} \in A$. Since $A$ is even affine, $\mathbf{p}^{*}$ is also the unique solution to the normal equations

$$
\left\langle\mathbf{S}-\mathbf{p}^{*}, \mathbf{p}-\mathbf{p}^{*}\right\rangle_{\mathbf{v}}=0
$$

with $\mathbf{p} \in A$ being arbitrary. Using the definition of the inner product $\langle., .\rangle_{\mathbf{V}}$, the normal equations can also be written as

$$
\left(\boldsymbol{v}-\mathbf{p}^{*}\right)^{\prime} \mathbf{V}^{-1}\left(\mathbf{p}-\mathbf{p}^{*}\right)=0
$$

We now observe that every vector $\mathbf{q}_{\gamma}:=\boldsymbol{v}+\gamma \mathbf{V} \mathbf{1}$ with $\gamma \in \mathbb{R}$ satisfies

$$
\left(\boldsymbol{v}-\mathbf{q}_{\gamma}\right)^{\prime} \mathbf{V}^{-1}\left(\mathbf{p}-\mathbf{q}_{\gamma}\right)=-\gamma\left(\mathbf{1}^{\prime} \mathbf{p}-\mathbf{1}^{\prime} \mathbf{q}_{\gamma}\right)
$$

and that $\mathbf{q}_{\gamma} \in A$ if and only if $\gamma=t\left(\mathbf{1}^{\prime} \mathbf{V} \mathbf{1}\right)^{-1}$. We have thus shown that the vector $\mathbf{q}:=\boldsymbol{v}+t\left(\mathbf{1}^{\prime} \mathbf{V} \mathbf{1}\right)^{-1} \mathbf{V} \mathbf{1}$ satisfies $\mathbf{q} \in A$ and

$$
(\boldsymbol{v}-\mathbf{q})^{\prime} \mathbf{V}^{-1}(\mathbf{p}-\mathbf{q})=0
$$

for all $\mathbf{p} \in A$. Therefore, we have $\mathbf{q}=\mathbf{p}^{*}$.

\section{REFERENCES}

De Vylder, E.F. (1996) Advanced Risk Theory. Bruxelles: Editions de l'Université de Bruxelles. Swartz, C. (1994) Measure, Integration and Function Spaces. New Jersey - London: World Scientific.

ZAKs, Y., Frosting, E., and LeviKson, B. (2006) Optimal pricing of a heterogeneous portfolio for a given risk level. ASTIN Bulletin 36, 161-185.

KLAUS D. SCHMIDT

Lehrstuhl für Versicherungsmathematik

Technische Universität Dresden

D-01062 Dresden

E-mail: klaus.d.schmidt@tu-dresden.de 